\title{
The intestinal epithelium compensates for p53- mediated cell death and guarantees organismal survival
}

\author{
YA Valentin-Vega ${ }^{1}$, H Okano² and G Lozano ${ }^{*, 1}$
}

Mdm2 is the major inhibitor of the p53 tumor suppressor. Loss of Mdm2 in mice or in specific tissues of the mouse always yields p53-dependent lethal phenotypes. However, the role of Mdm2 in tissues with high turnover capacity is unknown. We have engineered mice lacking Mdm2 in the intestinal epithelium using the Cre/LoxP system. Loss of Mdm2 (Mdm2 $\left.{ }^{\text {int }}\right)$ results in viable animals, but neonates display multiple intestinal abnormalities such as hyperplasia, enterocyte vacuolization, and inflammation. These defects correlate with a drastic increase in p53-dependent apoptosis in highly proliferative and differentiated cells. Unexpectedly, the observed phenotypes disappear with age. The tissue selects against Mdm2-null cells and increases its proliferative capacity. Additionally, the intestinal stem and progenitor cell populations are enriched leading to an increase in crypt fission events. Enhanced proliferation is achieved by activation of the canonical Wnt and EGFR-mediated Ras/MAPK pathways. While Mdm2 is a critical inhibitor of p53 in the intestinal epithelium, the tissue employs a series of processes that compensate for cell death.

Cell Death and Differentiation (2008) 15, 1772-1781; doi:10.1038/cdd.2008.109; published online 18 July 2008

The proliferative capacity of cancer cells is the result of accumulation of many genetic changes. A key alteration is mutation of the p53 tumor suppressor, which acts as a transcription factor to activate genes involved in apoptosis, cell-cycle arrest, and senescence. ${ }^{1}$ Disruption of p53 activity, as occurs in human tumors, therefore promotes cell proliferation and increased tumorigenesis.

The strong antiproliferative activity of p53 not only prevents outgrowth of malignant cells, but also has detrimental effects in normal cells when activated inappropriately. Numerous p53-negative regulators have been discovered that dampen p53 activity in normal cells. ${ }^{2}$ Among these, Mdm2 plays a central role in restricting p53 function.

Many tumors with high Mdm2 levels retain wild-type p53. ${ }^{3-6}$ Mdm2 is an E3 ubiquitin ligase that targets p53 for proteasome-dependent degradation. ${ }^{7-10}$ In mice, loss of Mdm2 results in embryo lethality. ${ }^{11-13}$ p53 deletion completely rescues this phenotype and demonstrates that Mdm2 is a critical inhibitor of p53 in vivo. Studies to elucidate tissuespecific functions of Mdm2 show that mice that express about $30 \%$ of the total Mdm2 protein levels display a decreased body weight, show defects in hematopoiesis, and are more radiosensitive than control animals due to increased p53mediated apoptosis. ${ }^{14}$ Loss of Mdm2 in cells of the central nervous system or cardiomyocytes results in embryonic lethality due to p53-dependent apoptosis. ${ }^{15-17}$ Also, deletion of Mdm2 specifically in smooth muscle cells or in osteoblasts show p53-mediated lethality. ${ }^{18,19}$ These studies confirm that Mdm2 is a potent inhibitor of p53 in a variety of specialized cells. Although the function of Mdm2 has been elucidated in the aforementioned cell types, it remains unknown how Mdm2 modulates p53 activity in tissues with high cellular turnover. We, therefore, examined the role of Mdm2 in the intestinal epithelium as it is composed of both highly proliferative and terminally differentiated cells.

The murine intestinal epithelium differentiates into a singlelayered epithelium around 14.5 days of gestation. ${ }^{20}$ Soon after, this layer invaginates to form intervillus pockets that contain stem and progenitor cells. Terminally differentiated cells become restricted to finger-like protrusions known as villi. Around postnatal day 7 (P7), rudimentary crypts of Lieberkühn start to emerge from the intervillus pockets and become the proliferative region. ${ }^{21}$ Cells in the crypt region migrate upward toward the villus compartment and become terminally differentiated. Finally, 2-7 days after leaving the crypts, mature epithelial cells are sloughed into the intestinal lumen. ${ }^{22}$ Thus, the intestinal epithelium represents the tissue with the fastest cellular turnover.

p53 plays an important role in the homeostasis of the intestinal epithelium during DNA damage. Undifferentiated cells at the base of the crypts undergo p53-dependent apoptosis in response to ionizing radiation, ${ }^{23-25}$ while more differentiated cells undergo cell-cycle arrest. $^{25}$ Thus, $^{2}$ undifferentiated epithelial cells appear more sensitive to

${ }^{1}$ Department of Genetics, Program in Genes and Development, The Graduate School of Biomedical Sciences, University of Texas MD Anderson Cancer Center, Houston, TX, USA and ${ }^{2}$ Department of Physiology, Keio University School of Medicine, Shinjuku-ku, Tokyo, Japan

*Corresponding author: G Lozano, Department of Genetics, The University of Texas MD Anderson Cancer Center, 1515 Holcombe Blvd., Unit 1010, Houston, TX 77030 , USA. Tel: + 713834 6386; Fax: + 7138346380 ;

E-mail: gglozano@mdanderson.org

Keywords: intestinal stem cells; $\beta$-catenin; EGFR; Ras/MAPK; Musashi-1

Abbreviations: ERK1/2, extracellular signal-regulated kinases 1 and 2; FM, Floxed $M d m 2$ allele; MAPK, mitogen-activated protein kinase; $M d m 2^{\text {int }}$, recombined Mdm2 allele; Msi-1, musashi 1; N, number of experimental animals; P3, postnatal day 3; VilCre, Villin Cre transgene

Received 22.5.08; revised 17.6.08; accepted 17.6.08; Edited by J-C Marine; published online 18.7 .08 
p53-mediated apoptosis, while differentiating epithelial cells are prone to p53-dependent cell-cycle arrest in response to DNA damage. To understand the in vivo significance of Mdm2 in regulating p53 activity in highly proliferative tissues, we implemented the Cre-LoxP strategy to delete $M d m 2$ in the intestinal epithelium. We found that Mdm2 loss leads to apoptosis in all compartments of the epithelium. With time, however, the tissue implements a variety of mechanisms to compensate for cell loss.

\section{Results}

Mice survive Mdm2 loss in the intestinal epithelium but display severe intestinal abnormalities. Loss of Mdm2 in mice causes embryo lethality. ${ }^{11-13}$ Therefore, conditional deletion of $M d m 2$ is required to assess the role of $M d m 2$ in more specialized cells. A loxP-flanked Mdm2 allele $\left(M d m 2^{F M}\right)$ generated in our laboratory phenocopies the original Mdm2-null allele upon Cre recombination. ${ }^{12,26}$ To address the in vivo role of $M d m 2$ in regulating p53 activity in tissues with high turnover capacity, we generated mice lacking Mdm2 exclusively in the intestinal epithelium using the Villin Cre (VilCre) transgene. ${ }^{27}$ We verified the specificity and efficiency of VilCre in targeting intestinal epithelial cells by mating mice carrying this transgene to the ROSA26R reporter, ${ }^{28}$ and performing $\beta$-galactosidase assays. Strong and specific $\beta$-galacotsidase activity was detected in almost all intestinal epithelial cells at embryonic day 14.5 (E14.5) and at 8 weeks (Supplementary Figure 1).

To ablate Mdm2 in intestinal epithelial cells, we generated $M d m 2^{F M /-}$ VilCre + and $M d m 2^{F M / F M}$ VilCre+ mice. Importantly, Mdm2 mutants of both genotypes displayed comparable phenotypes. Therefore, to simplify nomenclature, we labeled $M d m 2^{F M / F M}$ VilCre + or $M d m 2^{F M /-}$ VilCre + mice as $M d m 2^{i n t s}$. Analysis of progeny at weaning indicated that mice lacking $M d m 2$ in the intestinal epithelium survive at the expected ratio (Supplementary Table 1). We observed the recombination in cells from small intestines and colons, but not in tails, of $M d m 2^{\text {ints }}$ mice at 3 days of age (Figure 1a). The nonrecombined alleles detected are derived from either nonrecombined epithelium or other cell types present in the sample.

Characterization of $M d m 2^{\text {ints }}$ mice showed that by postnatal day 3 (P3), these mice displayed a significant reduction in body weight relative to control littermates (Figure $1 \mathrm{~b}$; control, $2.3 \mathrm{~g} \pm 0.035 ; \quad M d m 2^{\text {ints }}, 1.67 \mathrm{~g} \pm 0.059 ; \quad t$-test; $P<0.001 ; n \geqslant 23$ per group). The presence of milk in the stomachs of small Mdm2 $2^{\text {int }}$ mice suggests that the loss of body weight was not due to an inability to obtain milk (data not shown). Sections through the intestinal tract at P3 revealed that $M d m 2^{i n t}$ pups exhibited abnormal morphology in intervillus pockets as well as in villi (Figure 1c and d). Notably, smaller $M d m 2^{i n t s}$ pups showed a more severe phenotype than $M d m 2^{\text {ints }}$ pups of normal size and weight. The intervillus pockets of P3 Mdm2 ints mice displayed hypertrophy, hyperplasia, disorganization, and loss of polarity. Additionally, these pockets were significantly wider in mutant pups relative to controls (Figure $1 \mathrm{e}$ and $\mathrm{f}$; control, $1 \pm 0.08 ; \mathrm{Mdm}^{\text {ints }}$, $1.79 \pm 0.22$; $t$-test, $P=0.004)$. On the other hand, the villus region of P3 Mdm2 $2^{\text {ints }}$ pups showed atrophy and frequently displayed abnormal structures that appear to be vacuoles (Figure 1g). P3 Mdm2 $2^{\text {ints }}$ pups occasionally showed edema and neutrophil infiltration into the mucosa, indicative of inflammation (Figure 1d, and data not shown). Morphological analysis during embryogenesis revealed the absence of vacuoles in $M d m 2^{\text {ints }}$ intestines (Supplementary Figure 2), suggesting that the vacuoles observed at P3 are caused by inflammation. Staining using differentiation-specific markers to distinguish enterocyte, goblet, and enteroendocrine cells (Paneth cells are absent at this developmental stage) revealed the presence of all cell types in $M d m 2^{\text {ints }}$ intestine (Supplementary Figure 3a-d). Villin, alkaline phosphatase, alcian blue, and synaptophysin staining was similar in intestines of P3 Mdm2 $2^{\text {int }}$ pups and controls (Supplementary Figure 3). The aforementioned phenotypes were never observed in mice that carried one wild-type Mdm2 allele with or without VilCre+.

Mdm2-modulated p53 activity in highly proliferative and terminally differentiated cells of the intestinal epithelium. As Mdm2 is an E3-ubiquitin ligase that targets p53 for proteosome-mediated degradation, ${ }^{7-10}$ we tested whether the loss of Mdm2 in cells results in p53 stabilization. ${ }^{15-18}$ As predicted, P3 Mdm2 $2^{\text {ints }}$ pups displayed high levels of p53 protein in intestinal epithelial cells, while control pups lacked p53 expression (Figure 2a). High p53 levels were observed in highly proliferative cells of the intervillus pockets and in terminally differentiated cells in the villus region (Figure 2a). In general, smaller mice showed more p53-positive cells. These data reaffirm the absence of $M d m 2$ in the intestinal epithelium of $M d m 2^{\text {ints }}$ neonates.

Loss of Mdm2 in the mouse induces p53-mediated apoptosis at E3.5. ${ }^{11-13} \mathrm{We}$, therefore, examined the intestines of small ( $\leqslant 1.5 \mathrm{~g}$ ) P3 Mdm2 $2^{\text {ints }}$ pups for apoptosis. Loss of $M d m 2$ triggered a large increase in the number of apoptotic cells in the intervillus region (Figure $2 \mathrm{~b}$ and $\mathrm{c}$; control, $0.045 \pm 0.03 ; M d m 2^{\text {ints }}, 2.50 \pm 0.20 ; t$-test, $\left.P=0.002\right)$. A significant number of apoptotic cells were also present in intestinal villi of P3 Mdm2 $2^{\text {int }}$ mice (Figure 2b; control, $0.06 \pm 0.08 ; M d m 2^{\text {ints }}, 0.90 \pm 0.01 ; t$-test, $\left.P=0.005\right)$. Finally, P3 Mdm2 $2^{\text {ints }}$ pups displayed numerous apoptotic cells in the large intestine, emphasizing the importance of Mdm2 in this region as well (data not shown). Quantitative RNA analysis indicated that the apoptotic p53-target genes PUMA and $B A X$, but not PERP and NOXA, were significantly upregulated in $M d m 2^{\text {ints }}$ pups compared to controls (Figure 2d; $t$-test, $P<0.02, n=3-5$ per group). Although cell-cycle arrest was not observed in Mdm2 ints mice (see below), the p53-target genes, p21 and cyclin G1, were also upregulated in mutant intestines (Figure 2d and Supplementary Figure 4). Lastly, the increase in apoptosis and morphological defects observed in $M d m 2^{\text {int } 4}$ pups were completely rescued upon deletion of $p 53$ (Figure 2c and e). Collectively, these data indicate that Mdm2 is a major regulator of p53 stability and activity in both proliferative and terminally differentiated cells of the intestinal epithelium.

Intestinal epithelium compensated for cellular loss in the absence of Mdm2. To further explore the mechanisms 


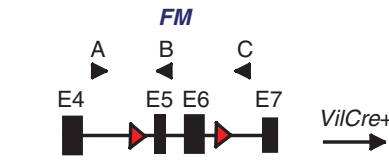

$2.3 \mathrm{~kb}$

$474 \mathrm{bp}$

b
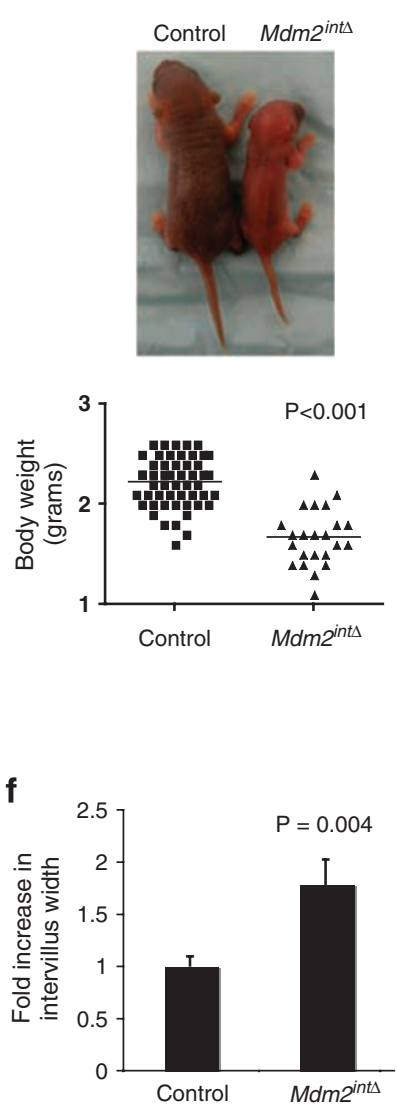
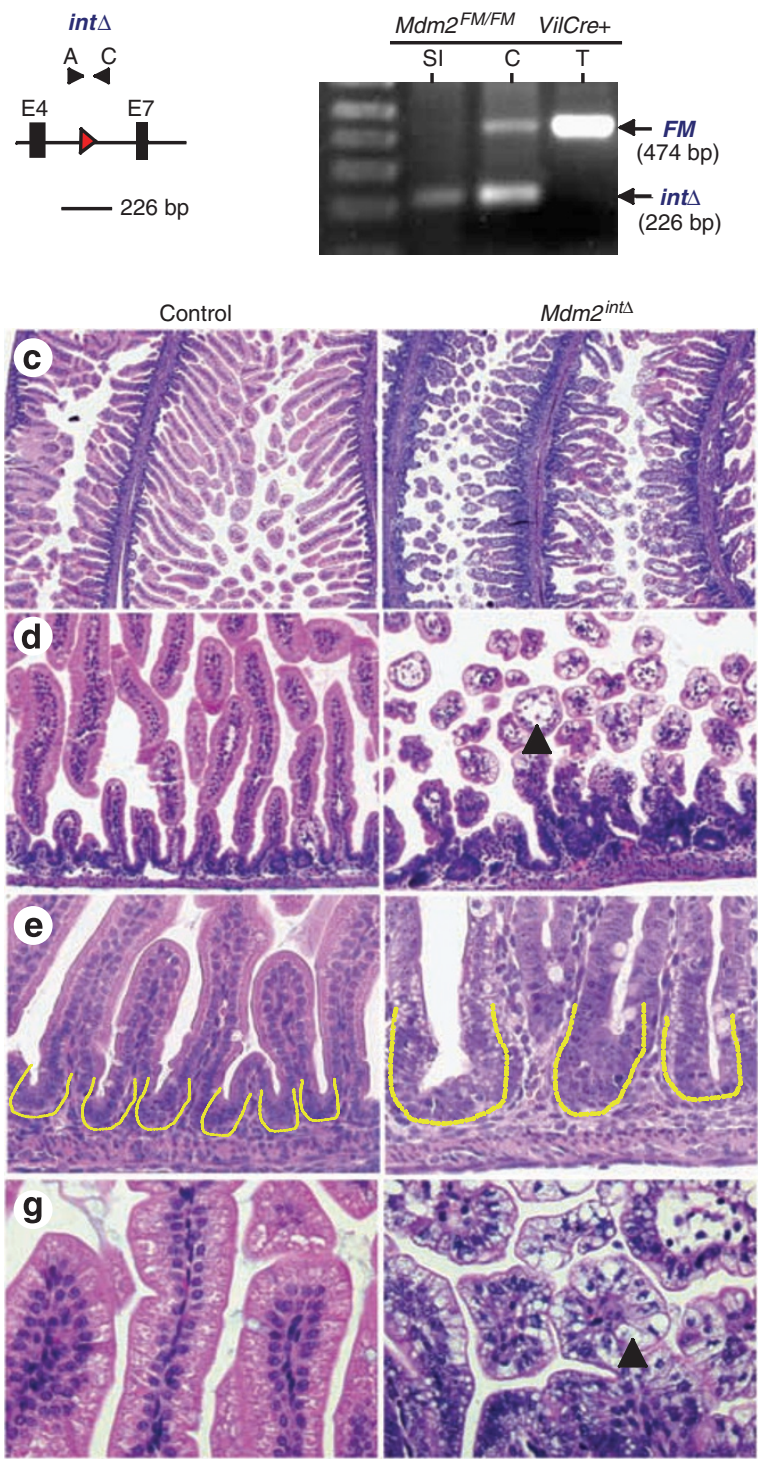

Figure 1 Young Mdm2 $2^{\text {ints }}$ mice developed multiple intestinal abnormalities. (a) PCR analysis of P3 pups show the nonrecombined floxed Mdm2 (FM) and recombined (int4) Mdm2 alleles. Red triangles, loxP sites; black triangles, primers; black lines indicate PCR products. E, exon; kb, kilobases, bp, base pairs. SI, small intestine; C, colon; T, tail. (b) Body size and weight of P3 littermates of different genotypes. (c and d) H\&E staining of small intestines of P3 mice at original magnification $\times 50$ (c) or $\times 200$ (d). Black arrowhead marks an intestinal edema in an $M d m 2^{\text {int }}$ pup. (e and f) Measurement of the intervillus width of P3 Mdm2 $2^{\text {int }}$ and control mice. Yellow lines denote the width of intervillus pockets. (g) Intestinal villi of P3 mice exhibiting vacuoles in enterocytes (black arrowhead)

by which $M d m 2^{\text {ints }}$ mice survive, we examined the phenotypes of adult mice. Adult $M d m 2^{\text {ints }}$ mice showed a normal intestinal morphology (Figure 3a). Adult $M d m 2^{\text {ints }}$ intestines showed only a few p53-positive cells in all regions of the small intestine and colon in contrast to the intestines of P3 $M d m 2^{\text {ints }}$ pups (compare Figure $2 \mathrm{a}$ versus Figure $3 \mathrm{~b}$ ). Moreover, adult $M d m 2^{\text {int }}$ mice showed a drastic reduction in the number of apoptotic cells in intestines relative to $\mathrm{P} 3$ $M d m 2^{\text {ints }}$ pups (Figure 3c; Mdm2 $2^{\text {ints }}$ at P3, 2.50 \pm 0.20 ; $M d m 2^{\text {ints }}$ at 8 weeks (jejunum), $0.595 \pm 0.035$; $t$-test, $P=0.007)$. This phenomenon was observed in the duodenum, jejunum, and ileum, and in the colon of adult mutant animals (data not shown). Lastly, P3 Mdm2 $2^{\text {ints }}$ pups with reduced body weight, gained weight as they aged. At 8 weeks of age, the body weight of these mutants showed no significant difference as compared to controls (Figure $3 d$ and data not shown). These data demonstrate that the intestinal epithelium is able to compensate for cell loss due to p53 activity.

The intestinal epithelium selected against cells with high p53 activity. A possible mechanism for compensation is that some intestinal cells in adult $M d m 2^{\text {ints }}$ mice retain $M d m 2$. To test this possibility, Mdm2 $2^{\text {ints }}$ mice carrying the $R O S A 26 R$ reporter were first analyzed for recombination at the ROSA26R locus. LacZ-stained sections showed uniform expression of VilCre throughout the epithelium in $R 26 R /+$ VilCre + P3 pups, indicating high efficiency of recombination (Figure 4a). In contrast, a chimeric pattern was observed in intestinal epithelial cells of P3 Mdm2 $2^{F M / F M} R 26 R /+$ VilCre+ 

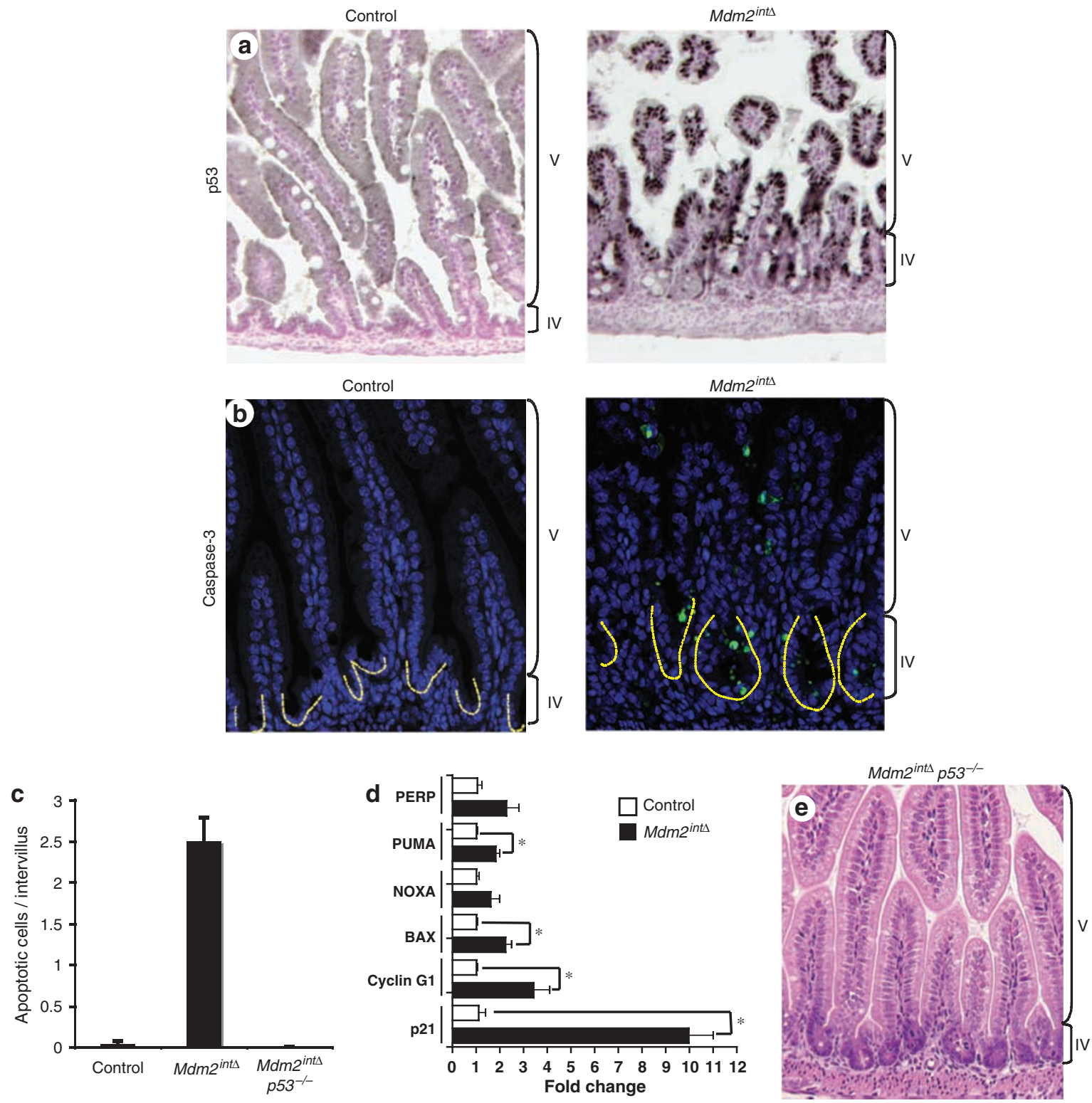

Figure 2 Mdm2 regulated p53 activity in highly proliferative and terminally differentiated intestinal epithelial cells. (a) Immunohistochemistry with p53 antibodies in small intestines of P3 mice. (b) Apoptotic cells were labeled by Caspase-3 immunofluorescence (green) and nuclei with TOPRO-3 (blue). Yellow lines outline intervillus pockets. (c) Quantification of apoptosis in intervillus pockets at $\mathrm{P} 3$ evaluated by scoring apoptotic bodies in H\&E-stained sections (200 intervillus units scored per mouse; $n \geqslant 3$ per group). (d) mRNA quantification of p53-target genes by reverse transcriptase real-time PCR analysis. ( $n \geqslant 3$ per group, asterisk means $P<0.02$ ). (e) H\&E-stained small intestines of P3 Mdm2 $2^{\text {ints }}$ neonates without $p 53$. V, villus region; IV, intervillus region

pups. Eight-week-old $M d m 2^{\text {ints }}$ mice showed a further decrease in recombination events in the intestinal epithelium; few patches were observed in the crypt compartment suggesting that most crypts have already lost the Cre recombinase (Figure $4 a$ ).

To investigate whether the loss of recombination also occurred at the Mdm2 locus with age, we analyzed the recombination using primer sets that distinguish the wild-type, Mdm2 conditional (FM), and recombined (int 4 ) alleles (Figure 1a), and DNA isolated from scraped intestinal mucosa of 8-week-old animals to enrich for an epithelial population. Control 8-week-old $\mathrm{Mdm} 2^{\mathrm{FM} /-}$ VilCre- mice did not have the recombined allele, while $\mathrm{Mdm} 2^{\mathrm{FM} /+} \mathrm{VilCre}+$ mice recombined the $M d m 2$ conditional allele with high efficiency (Figure 4b). In 8-week-old Mdm2 ${ }^{\mathrm{FM} /-}$ VilCre + mutants, the number of recombined cells was greatly reduced as compared to 8-week-old $\mathrm{Mdm2} 2^{\mathrm{FM} /+}$ VilCre + mice. As the 3-day-old $\mathrm{Mdm} 2^{\mathrm{FM} /-}$ VilCre + pups also show high efficiency of recombination events (Figure $4 b$ ), loss of recombination at the Mdm2 locus occurs with time. Together, these data suggest that the intestinal epithelium selects against cells that have high p53 activity.

A potential mechanism by which the intestinal epithelium selects against cells with high p53 activity is by loss of the VilCre transgene. To investigate this possibility, real-time PCR was performed using DNA from scraped intestinal 

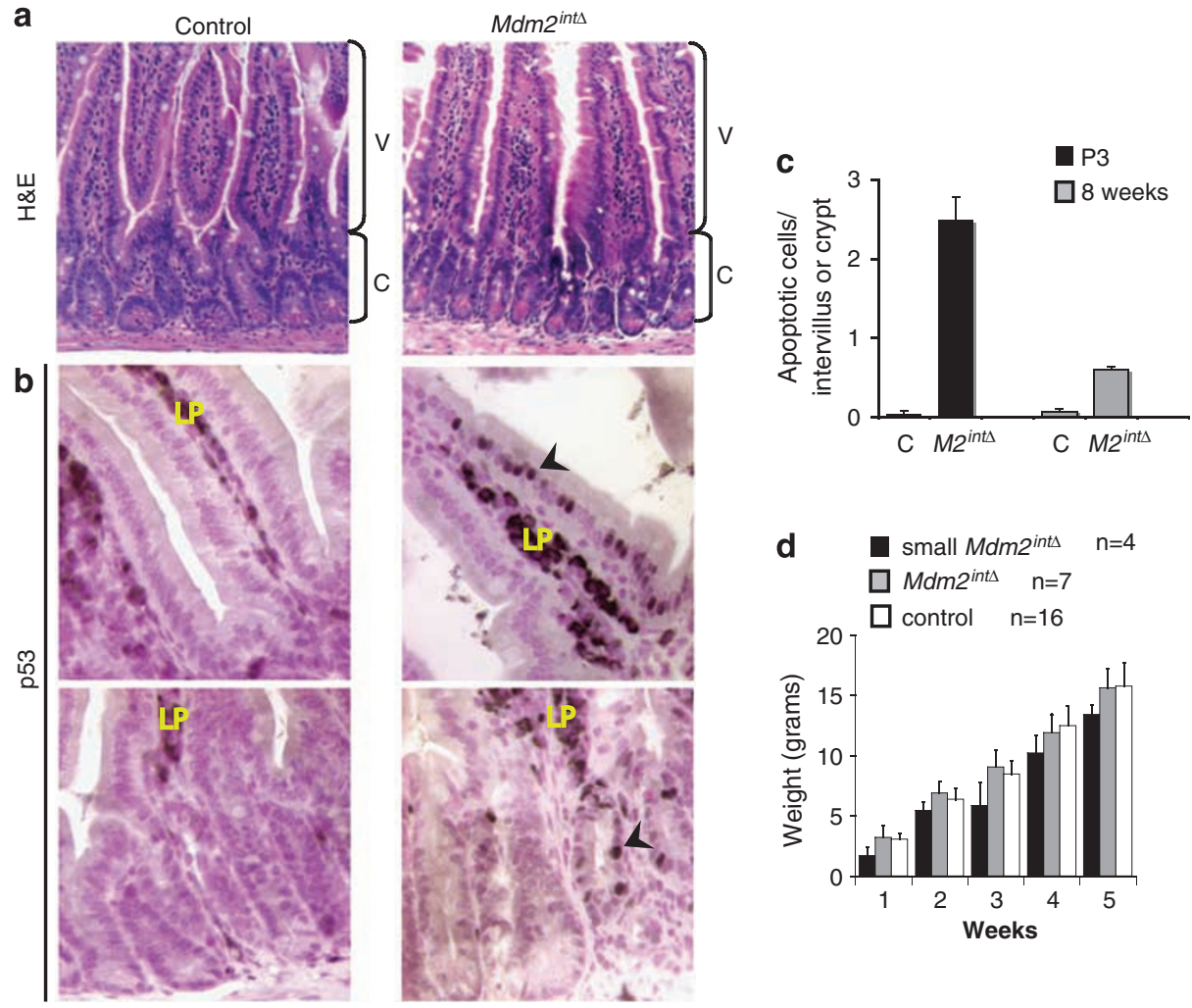

Figure 3 Intestines of Adult Mdm2 ${ }^{\text {int }}$ mice have decreased p53 levels and recover. (a) H\&E staining of intestinal jejunum of 8-week-old mice. V, villus region; C, crypt region. (b) p53 immunohistochemistry performed in intestinal jejunum of 8-week-old mice (original magnification $\times 600$ ). Staining in the lamina propria (LP) is an artifact. Black arrowheads denote p53-positive cells. (c) Comparison of the apoptotic indices between P3 (black bars) and 8-week-old (gray bars) Mdm2 ${ }^{\text {int }}$ mice. Apoptosis was measured by scoring apoptotic bodies in the jejunum of adult mice and in whole intestines of P3 mice (200 intervillus units scored per mouse; $n=3$ per group). C, control group, M2 ${ }^{\text {int }}$, $M d m 2^{\text {ints }}$ mice. (d) Body weight gain of control (white bars), normal size $M d m 2^{\text {ints }}$ (gray bars) and small size Mdm2 $2^{\text {int }}$ (black bars) mice by week

mucosa of 8-week-old mice. As control, we used $M d m 2^{F M /+}$ $\mathrm{VilCre}+$ mice. The 8-week-old $\mathrm{Mdm}^{\mathrm{FM} /-} \mathrm{VilCre}+$ mutants showed greater than 100-fold reduction in the total amount of the VilCre transgene in the small intestine relative to tail (Table 1). These data suggest that the intestinal epithelial cells from $M d m 2^{\text {ints }}$ mice spontaneously delete the VilCre transgene and thus are able to expand.

Multiple mechanisms compensated for p53 activation in the intestinal epithelium. To further elucidate the mechanisms of cellular compensation upon p53 activation, we investigated the proliferation capacity of the intestinal epithelium in $M d m 2^{\text {ints }}$ mice. P3 Mdm2 $2^{\text {ints }}$ mice showed a twofold increase in Ki-67-positive cells per intervillus unit as compared to control animals, indicating that intestines of these mutants have an accelerated proliferative capacity (Figure 5a; control, $10.43 \pm 0.189$; $M d m 2^{\text {int }}, 18.98 \pm 2.85$; $t$-test, $P=0.007)$. Moreover, $\mathrm{BrdU}$ assays performed in adult intestines showed that $M d m 2^{\text {int }}$ mice retained an increased capacity to proliferate relative to age-matched control animals (Supplementary Figure 5; control, $26.2 \pm 0.22$; $M d m 2^{\text {int }}, \quad 36.9 \pm 2.6 ; \quad t$-test, $\left.P=0.036\right)$. However, the proliferation index of the intestine was relatively less in adult mutants than in P3 mutants. Therefore, the intestinal epithelium compensates for $p 53$ activation by increasing cell proliferation.
The postnatal growth of the small intestine occurs through two major processes: crypt hyperplasia and crypt fission. ${ }^{29}$ Given that neonatal $M d m 2^{\text {ints }}$ mice recovered from the detrimental effects of p53 activation, we counted the number of crypt fissions. Indeed, P3 Mdm2 ints pups showed a drastic increase in the frequency of crypt fissions in the small intestine (Figure 5b; control, $1.225 \pm 0.64 ; \mathrm{Mdm}^{\text {int } 4}, 4.972 \pm 1.28$; $t$-test, $P=0.0001)$. Thus, another mechanism by which $M d m 2^{\text {ints }}$ mice survive is the ability to increase crypt fission events.

The crypt fission process is thought to depend on the multiplication of intestinal stem cells. ${ }^{30} \mathrm{We}$, therefore, performed immunohistochemistry using the intestinal stem and progenitor cell marker, Musashi-1 (Msi-1). ${ }^{31,32}$ Analysis of expression of Msi-1 in P3 Mdm2 ${ }^{\text {ints }}$ mice revealed more Msi1-positive cells in the small intestine of $M d m 2^{\text {ints }}$ pups than in control littermates (Figure 5c; control, 5.647 \pm 0.472 ; $M d m 2^{\text {ints }}, 9.338 \pm 0.614 ; t$-test, $\left.P<0.001\right)$. These results suggest that the intestinal stem and progenitor cell populations are increased in $M d m 2^{\text {int } t}$ neonates.

The canonical Wnt and EGFR-mediated Ras/MAPK signaling pathways were induced in the intestines of $\mathbf{M d m 2}^{\text {inta }}$ mice. The canonical Wnt pathway plays an important role in maintaining the proliferation capacity and stem-cell population of the intestinal epithelium. ${ }^{33}$ The 

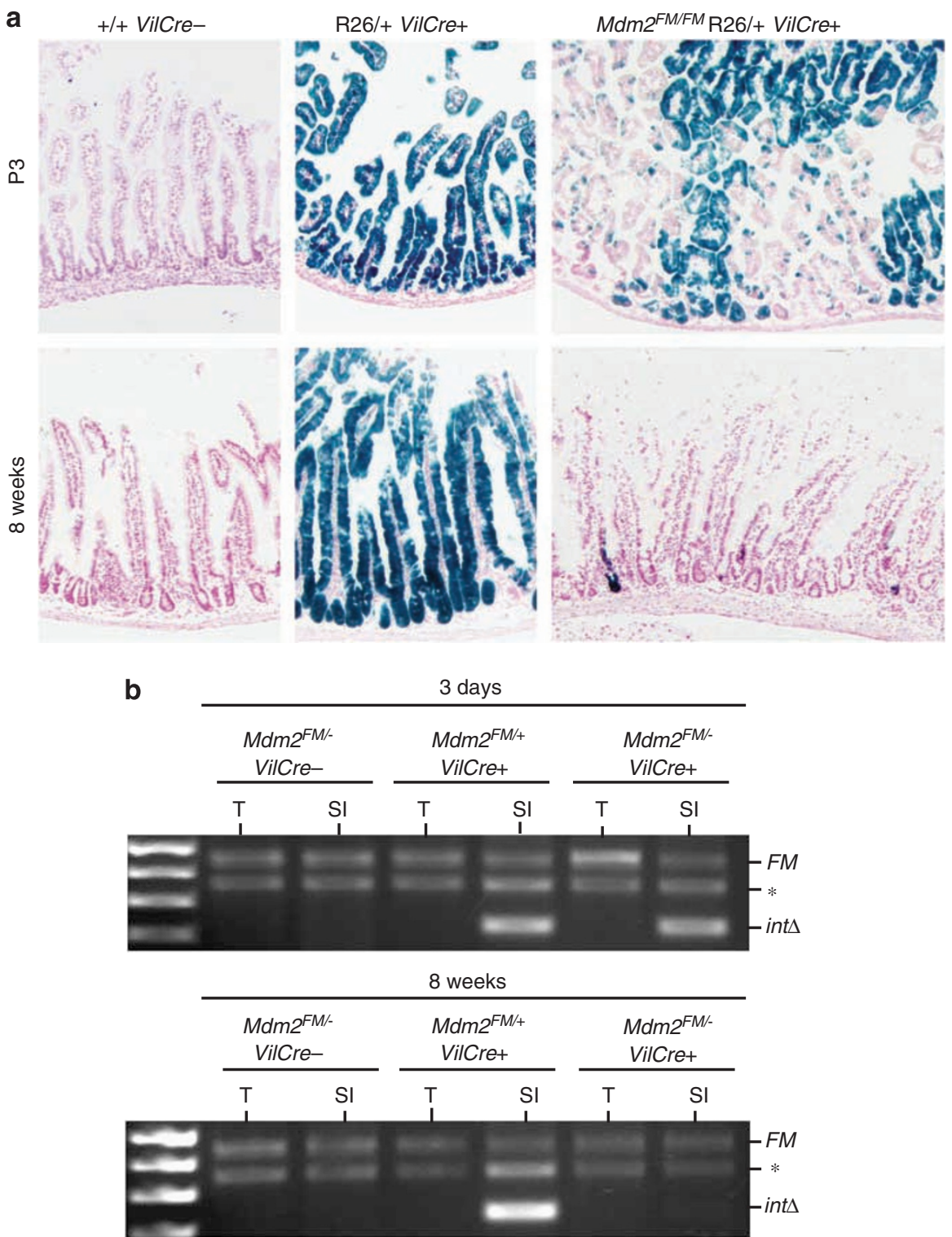

Figure 4 Intestines of $M d m 2^{\text {ints }}$ mice select against Mdm2-null cells. (a) $\beta$-galactosidase staining (blue) of small intestines of P3 and 8-week-old mice. (b) PCR-based recombination analysis in 3-day and 8-week-old mouse tissues ( $n \geqslant 3$ per group) using primers $A, B$, and C (see Figure 1a). Asterisk marks the band amplified using primers $A$ and $\mathrm{B}$, which represents the wild-type or Mdm2- null alleles. SI, small intestine; T, tail

Table 1 VilCre Tg DNA levels in small intestines of 8-week-old mice

\begin{tabular}{|c|c|c|}
\hline Mouse number & Genotype & Fold decrease $\mathrm{e}^{\mathrm{a}}$ \\
\hline $\begin{array}{l}\text { Control \#1 } \\
\text { Control \#2 } \\
M d m 2^{\text {ints }} \# 1 \\
M d m 2^{\text {ints }} \# 2 \\
M d m 2^{\text {ints }} \# 3\end{array}$ & $\begin{array}{l}\text { Mdm2 } 2^{F M /+} \text { VilCre+ } \\
M d m 2^{F M /+} \text { VilCre+ } \\
M d m 2^{F M /-} \text { VilCre+ } \\
M d m 2^{F M /-} \text { VilCre+ } \\
M d m 2^{F M /-} \text { VilCre+ }\end{array}$ & $\begin{array}{c}1.75 \\
6.6 \\
137 \\
1385 \\
9274\end{array}$ \\
\hline
\end{tabular}

asamples were normalized using GAPDH DNA as an internal control and compared to tail DNA

central player of this signaling pathway is $\beta$-catenin. Because $M d m 2^{\text {ints }}$ mice exhibited increased proliferative capacity and an increase in stem cells, we next tested the hypothesis that the canonical Wnt pathway is active in these mutants. P3 $M d m 2^{\text {ints }}$ pups had a larger number of cells in the intervillus compartment that expressed intracellular $\beta$-catenin as compared to controls (Figure 6a). To determine whether $\beta$-catenin was active in the intestine of neonatal $M d m 2^{\text {ints }}$ mice, we performed immunohistochemistry against CD44, a downstream target of the $\beta$-catenin/TCF-4 complex. ${ }^{34}$ Small intestines of P3 Mdm2 $2^{\text {int }}$ pups displayed drastic accumulation of this membrane glycoprotein in intervillus pockets as compared to controls (Figure 6b). Together, these data imply that the $\beta$-catenin/TCF- 4 complex is active in the intestinal epithelium of P3 Mdm2 ${ }^{\text {int }}$ pups.

To investigate other pathways that may be involved in tissue compensation in the absence of Mdm2, we performed gene expression profiles of RNA isolated from whole small 

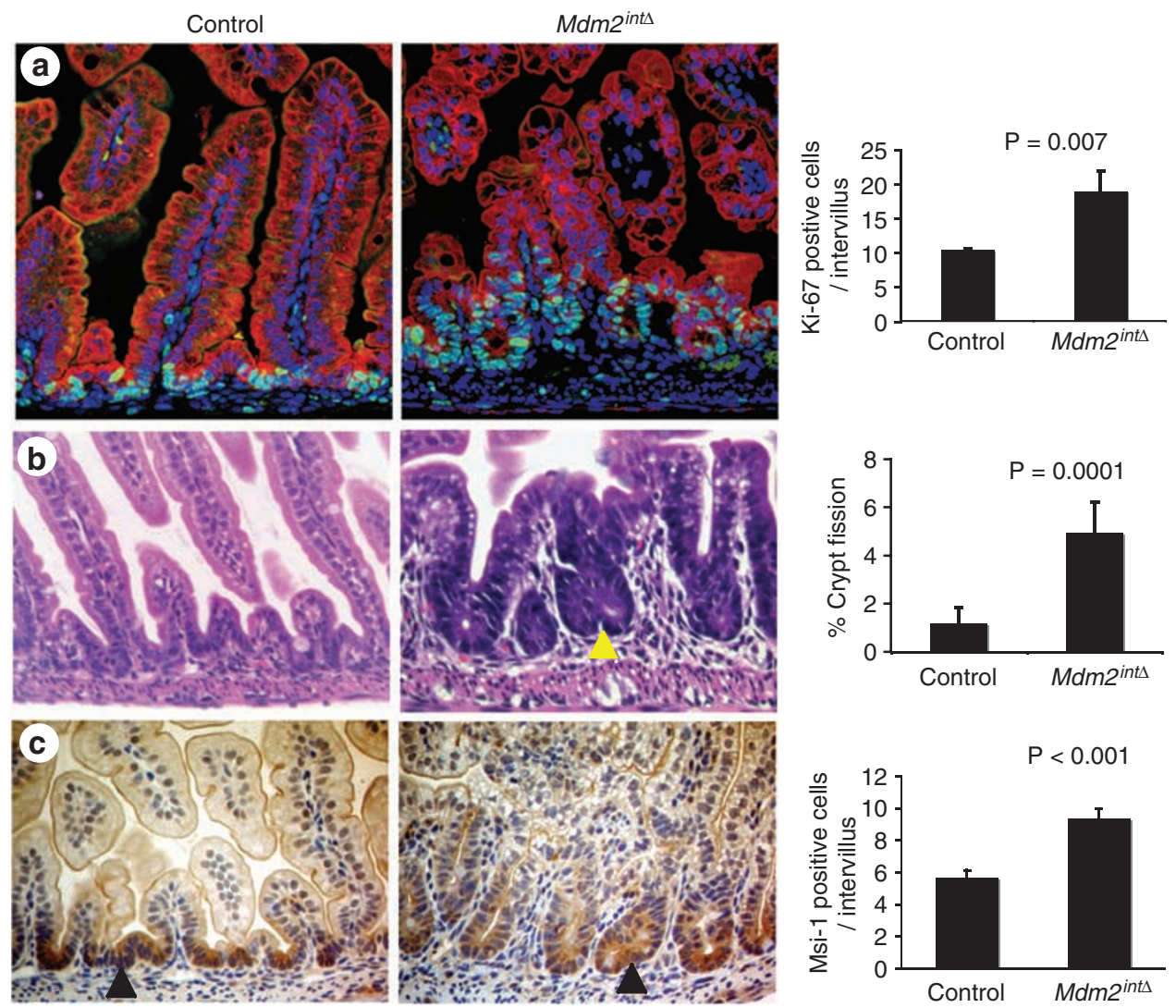

Figure 5 Mechanisms of intestinal compensation in Mdm2 $2^{\text {ints }}$ mice. (a) Ki-67 immunofluorescence (turquoise) in small intestines of P3 mice with cytokeratin (orange) and TOPRO-3 nuclear (dark blue; original magnification $\times 400$ ). Graph represents the quantification of the Ki-67 staining (200 intervillus units scored per mouse; $n=3$ per group). (b) H\&E staining and quantification of intervillus units of P3 mice (200 intervillus units scored per mouse; $n \geqslant 4$ per group; original magnification $\times 400$ ). Arrowhead pinpoints a crypt fission event occurring in an Mdm2 $2^{\text {ints }}$ pup. (c) Immunohistochemistry using an Msi-1antibody in intestines of P3 mice. Black arrowheads mark Msi-1-positive cells in intervillus pockets. A total of 200 intervillus units were scored per mouse (original magnification $\times 400, n \geqslant 4$ per group). Note that the pictures of both control and mutant intestines were taken at the same magnification, but the mutant intestines are larger due to the hyperplastic and hyperthrophic phenotype observed in these mutants

intestines of small Mdm2 $2^{\text {int }}$ pups by genome-wide microarray analyses. We identified 219 genes that showed at least a twofold change and exhibited a $P$-value of less than 0.05 and were either upregulated (139 genes) or downregulated (80 genes) in the intestine of $M d m 2^{\text {ints }}$ neonates as compared to control mice (Supplementary Table 2). Many of these genes are involved in immune response, xenobiotic and lipid metabolism, and cellular trafficking, suggesting that these processes are altered in the intestine of $M d m 2^{\text {ints }}$ mutants. The fact that these mutants show the loss of body weight supports this hypothesis. Notably, several well-known p53target genes were upregulated in the intestine of $M d m 2^{\text {ints }}$ neonates, confirming that these mutants display high p53 activity (Supplementary Table 2). The activation of some of these genes (i.e., p21, cyclin G1) was validated in these mutants (Figure 2d). Lastly, we detected a cluster of upregulated genes in the mutant intestines that have important functions in signal transduction such as amphiregulin, epiregulin, calcyclin, and augmenter of liver regeneration (ALR; Supplementary Table 2). Amphiregulin and Epiregulin are members of the epidermal growth factor family (known as EGF-like factors) that bind to EGF receptors (EGFR) and initiate a signal transduction cascade that activates pathways such as the Ras-activated, mitogenactivated protein kinase (MAPK) pathway (Figure 6c). ${ }^{35}$ Therefore, we postulated that the EGFR-mediated Ras/ MAPK pathway might play an important role during the tissue regeneration in response to p53 activation. To test this hypothesis, we first performed reverse transcription real-time PCR to analyze the mRNA expression of amphiregulin and epiregulin in P3 Mdm2 $2^{\text {int }}$ pups. We detected significantly higher mRNA levels of both amphiregulin and epiregulin in the intestines of $M d m 2^{\text {ints }}$ pups than in the intestines of control mice (Figure 6d; amphiregulin, control $=1.107 \pm 0.3095$, $M d m 2^{\text {ints }}=3.906 \pm 0.8370 \quad(P=0.0095, \quad n \geqslant 4$ per group $)$; epiregulin, control $=1.009 \pm 0.09861, M d m 2^{\text {int }}=5.716 \pm 0.9555$ $(P=0.008, n \geqslant 3$ per group)). Immunohistochemistry revealed that the intestines of P3 Mdm2 $2^{\text {int }}$ pups show increased levels of EGFR along the entire intervillus-villus axis, whereas control animals did not stain for EGFR (Figure 6e). Moreover, intestines of P3 Mdm2 $2^{\text {ints }}$ pups showed a dramatic increase in the expression of phosphorylated-ERK $1 / 2$ in the intervillus region as compared to controls (Figure 6f). Collectively, these data indicate that, like the canonical Wnt pathway, the EGFR-mediated Ras/MAPK pathway is active in the intestinal epithelium of P3 Mdm2 ${ }^{\text {ints }}$ pups. 
a
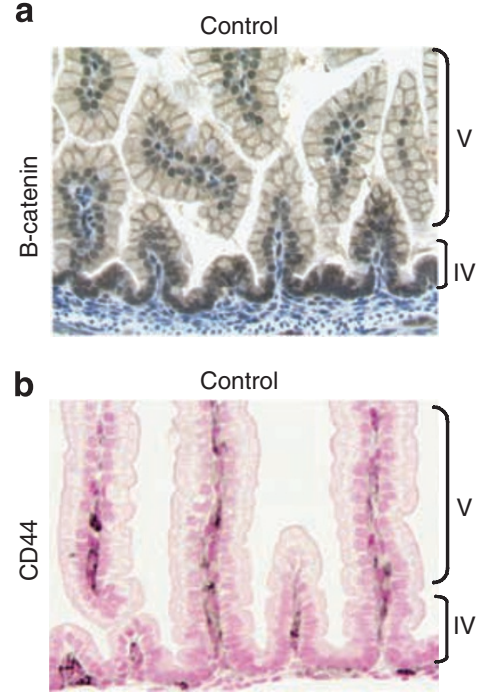
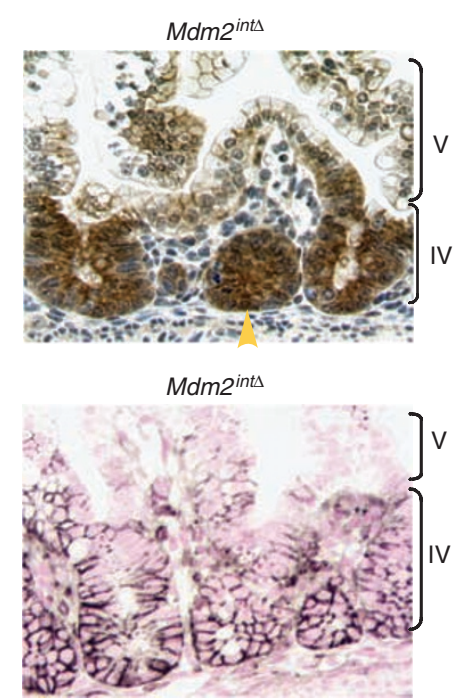
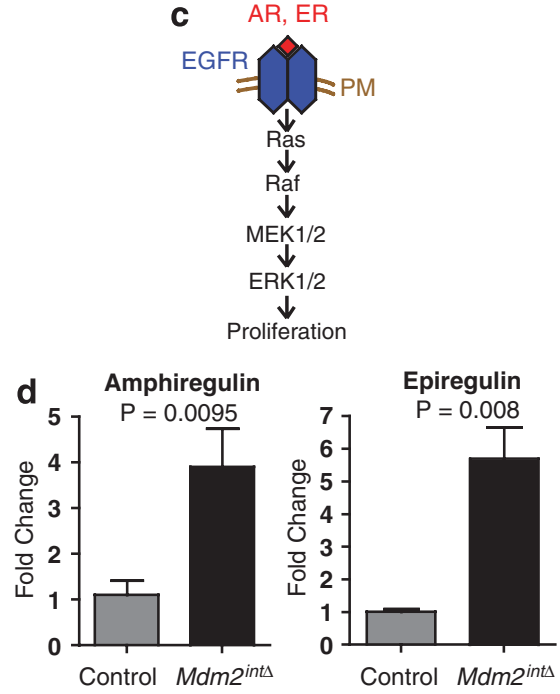
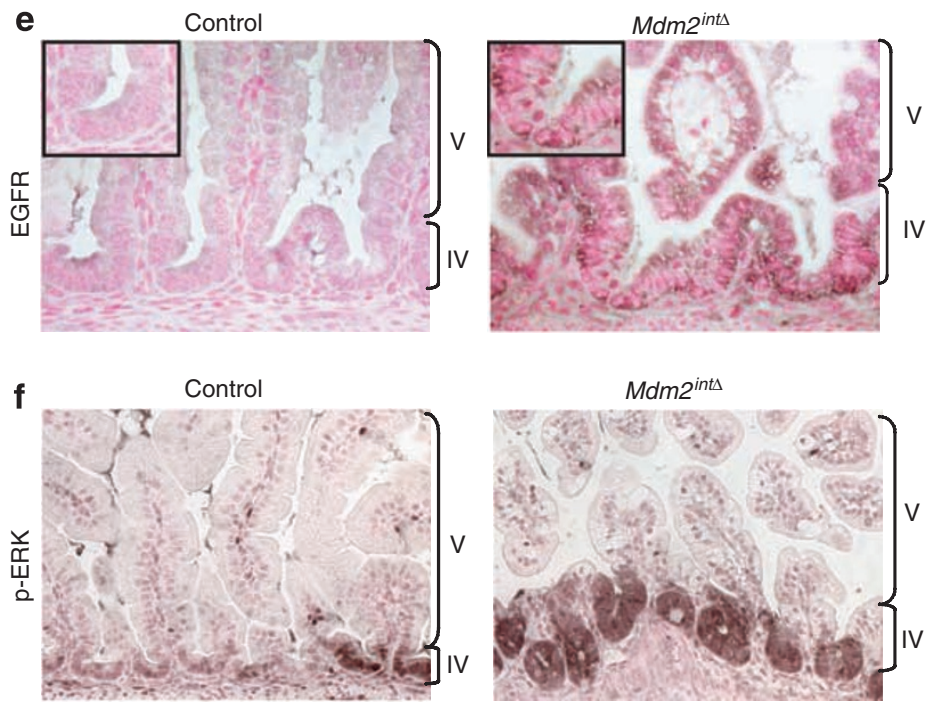

Figure 6 The canonical Wnt signaling, and EGFR-mediated Ras/MAPK pathways are active in the intestinal epithelium of Mdm2 $2^{\text {int }}$ neonates. (a) Immunohistochemistry against active $\beta$-catenin. Arrowhead pinpoints intracellular accumulation of $\beta$-catenin in the intervillus region of $M d m 2^{\text {ints }}$ neonates. (b) Immunohistochemistry against CD44, a membrane protein, in the small intestine of P3 mice. (c) The EGFR-mediated Ras/MAPK signaling cascade. Amphiregulin (AR) or Epiregulin (ER) bind to EGFR at the plasma membrane (PM) and induce Ras activity. Ras binds and activates Raf, which in turn phosphorylates and activates MEK1 and MEK2. Finally, MEK1/2 phosphorylates ERK1 and ERK2, which accumulate in the nucleus. In the nucleus, ERK1/2 phosphorylates a number of transcription factors involved in cell proliferation and survival. (d) Quantification of amphiregulin and epiregulin mRNAs in 3-day-old intestines ( $n \geqslant 3$ per group). (e) Immunohistochemistry against EGFR in P3 mouse intestines. Box shows higher magnification of the intervillus region. (f) Immunohistochemistry against p-ERK1/2 in 3-day-old small intestines. V, villus region, IV, intervillus region

\section{Discussion}

The data demonstrate that Mdm2 is a critical inhibitor of p53 activity in the intestinal epithelium. Absence of $M d m 2$ in this tissue results in an accumulation of p53 protein and enhanced p53-mediated apoptosis in both highly proliferative and terminally differentiated cells. Thus, p53 activation results in severe intestinal abnormalities early in life. The presence of apoptotic cells in differentiated intestinal epithelia with loss of Mdm2 was unexpected as earlier studies revealed that p53-mediated apoptosis is a rare event in this cell type under normal conditions or upon DNA damage. ${ }^{36}$ Therefore, these early studies show that sufficient Mdm2 levels in terminally differentiated intestinal epithelial cells keep p53 activity at bay in wild-type mice. The inhibition of p53 activity by Mdm2 also occurs in differentiated smooth muscle and brain cells. ${ }^{16,18}$ These data suggest that Mdm2 dampens p53 activity in differentiated cells despite the fact that they will never divide.

Other studies also indicate that the partial loss of Mdm2 increases apoptosis in the gut, ${ }^{37}$ but failed to note the other defects seen in our mice probably due to the presence of some Mdm2. Our Mdm2 $2^{\text {ints }}$ mice also exhibit inflammation, presence of vacuoles, and increase in intervillus width. Most importantly, increased apoptosis was observed in both, highly proliferative and terminally differentiated intestinal epithelial cells, a phenotype not observed in mice retaining some 
Mdm2. In mice expressing a tamoxifen inducible p53 allele, intestinal defects were also noted, although not characterized in detail. ${ }^{38}$

Although Mdm2 mutants exhibit numerous intestinal defects early in life, such defects disappear with age and these mice have a normal lifespan. The intestinal epithelium compensates for the detrimental effects of p53 activation through multiple mechanisms. First, intestines lacking Mdm2 are able to select against cells that are undergoing recombination through the loss of VilCre. A similar phenomenon was reported in mice with the deletion of c-Myc in intestinal crypts. ${ }^{39}$ Second, the tissue increases proliferation in young $M d m 2^{\text {ints }}$ mice to balance cell loss due to p53-dependent apoptosis. Third, the intestinal epithelium increases the number of crypt fission events upon Mdm2 deletion, which promotes intestinal growth. ${ }^{40}$ Fourth, the intestinal epithelium also compensates for p53 activation by increasing the putative stem-cell population. Thus, the increase in proliferation and crypt fission events is likely the result of an expanded stem-cell population. Thus, multiple mechanisms are coordinated to compensate for cell loss due to p53 activation.

Finally, the tissue senses the cellular abnormalities in the intestine and triggers the activation of the canonical Wnt, and EGFR-Ras/MAPK pathways, thus, promoting survival. The canonical Wnt/ $\beta$-catenin pathway is linked to intestinal stemcell maintenance and epithelial homeostasis. For example, loss of the $\beta$-catenin partner, TCF-4, in mice results in lethality due to complete absence of the proliferative compartment of the intestinal epithelium. ${ }^{41}$ On the other hand, the role of the EGFR-mediated Ras/MAPK pathway in intestinal regeneration is novel. In addition to their role in tissue homeostasis, these two pathways contribute to tumorigenesis. ${ }^{33,42}$ Thus, normal and neoplastic cells share similar molecular pathways that allow cell proliferation. However, normal cells cease to proliferate when the tissue reaches homeostasis, whereas tumor cells continue proliferating indefinitely. Despite the activation of the $\mathrm{Wnt} / \beta$-catenin and EGFR-mediated Ras/ MAPK pathways, Mdm2 $2^{\text {ints }}$ mice do not develop tumors. It would be of great interest to elucidate the molecular mechanisms normal cells employ to halt proliferation when the tissue reaches homeostasis.

\section{Materials and Methods}

Mice. All animal protocols were approved by the Institutional Animal Care and Use Committee. All mice were $>90 \% \mathrm{C} 57 \mathrm{BL} / 6$. VilCre mice were crossed with $M d m 2^{F M}$ conditional mice. ${ }^{27,26}$ Recombination was assayed by PCR using primers described previously. ${ }^{26}$ Intestines of P3 pups were washed in cold phosphate buffered saline (PBS), rolled, incubated in $10 \%$ formalin for up to $48 \mathrm{~h}$, and embedded in paraffin. Intestines of 8-week-old mice were cut into sections (duodenum, jejunum, ileum, and colon), and luminal contents flushed with cold PBS before rolling.

$\beta$-galactosidase activity assays. $\beta$-galactosidase activity in embryos was performed as described. ${ }^{15}$ Postnatal samples were fixed in $4 \%$ paraformaldehyde at $4^{\circ} \mathrm{C}$ for $1 \mathrm{~h}$. Samples were cryoprotected in a sucrose gradient (10\% sucrose/HBSS overnight; $15 \%$ sucrose/HBSS for $6 \mathrm{~h}$, and $20 \%$ sucrose/HBSS for $6 \mathrm{~h}$ ) at $4{ }^{\circ} \mathrm{C}$, embedded in optimum cutting temperature compound (Sakura), and $10 \mu \mathrm{m}$ sections cut at $-20^{\circ} \mathrm{C}$. Sections were fixed in $0.2 \%$ glutaraldehyde for $2 \mathrm{~min}$, washed in PBS (pH 8.3), and stained (1 mg/ml X-Gal, $50 \mathrm{mM} \mathrm{K}_{3} \mathrm{Fe}(\mathrm{CN})_{6}, 50 \mathrm{mM} \mathrm{K}_{4} \mathrm{Fe}\left(\mathrm{CN}_{6}\right)_{6} \cdot 3 \mathrm{H}_{2} \mathrm{O}$, $2 \% \mathrm{NP}-40,1 \%$ sodium desoxycholate, $1 \mathrm{M} \mathrm{MgCl}_{2}$ in PBS (pH 8.3)) overnight at $37^{\circ} \mathrm{C}$
Immunohistochemistry and immunofluorescence. The antibodies used for immunohistochemistry and immunofluorescence studies are listed in Supplementary Table 3. Immunohistochemistry was performed as described. ${ }^{3}$ For immunofluorescence, epitope retrieval was performed by steaming samples in $10 \mathrm{mM}$ citric acid (pH 6.0) for $30 \mathrm{~min}$. Samples were incubated in blocking serum (10\% goat serum in PBS- $0.1 \%$ Tween-20) for $1 \mathrm{~h}$ at room temperature, then with primary antibodies, followed by rinsing in PBS, and incubation with the fluorochrome-conjugated secondary antibody.

Microarray and reverse transcriptase real-time analyses. RNA was isolated from whole small intestines of P3 mice (RNAeasy kit, Qiagen). Five mice per group were analyzed individually using the Affymetrix GeneChip Mouse Genome 4302.0 Array. Student's t-test was used on log-base transformed data. Samples were considered statistically significant if $P$-values were less than 0.05 and showed at least twofold of change. For validation, RNA was reverse transcribed using the First-Strand cDNA Synthesis kit from Amersham Biosciences. Real-time was performed using the primer sets listed in Supplementary Table 4. Relative DNA levels were normalized to the value of GAPDH for each reaction.

Acknowledgements. This study was supported by NIH Grants CA47296 to GL and the Cancer Center Support Grant (CA16672). YAVV was supported by the Schissler and Sowell-Huggins Foundations. We thank Drs. Box, Xiong, Terzian, Henning for guidance, Drs. Kapoor and Zhang for microarray analyses, and Stephens for pathological analyses. Microarray was submitted to EBI ArrayExpress, accession number: E-MEXP-1431.

1. Vousden KH. p53: death star. Cell 2000; 103: 691-694.

2. Brooks CL, Gu W. p53 ubiquitination: Mdm2 and beyond. Mol Cell 2006; 21: 307-315.

3. Evans SC, Viswanathan M, Grier JD, Narayana M, El-Naggar AK, Lozano G. An alternatively spliced HDM2 product increases p53 activity by inhibiting HDM2. Oncogene 2001; 20: 4041-4049.

4. Momand J, Jung D, Wilczynski S, Niland J. The MDM2 gene amplification database. Nucleic Acids Res 1998; 26: 3453-3459.

5. Oliner JD, Kinzler KW, Meltzer PS, George DL, Vogelstein B. Amplification of a gene encoding a p53-associated protein in human sarcomas. Nature 1992; 358: 80-83.

6. Valentin-Vega YA, Barboza JA, Chau GP, El-Naggar AK, Lozano G. High levels of the p53 inhibitor MDM4 in head and neck squamous carcinomas. Hum Pathol 2007; 38 1553-1562.

7. Honda R, Tanaka H, Yasuda H. Oncoprotein MDM2 is a ubiquitin ligase E3 for tumor suppressor p53. FEBS Lett 1997; 420: 25-27.

8. Kubbutat MH, Jones SN, Vousden KH. Regulation of p53 stability by Mdm2. Nature 1997; 387: 299-303.

9. Momand J, Zambetti GP, Olson DC, George D, Levine AJ. The mdm-2 oncogene product forms a complex with the p53 protein and inhibits p53-mediated transactivation. Cell 1992; 69: $1237-1245$

10. Haupt $Y$, Maya R, Kazaz A, Oren M. Mdm2 promotes the rapid degradation of $p 53$. Nature 1997; 387: 296-299.

11. Jones SN, Roe AE, Donehower LA, Bradley A. Rescue of embryonic lethality in Mdm2-deficient mice by absence of p53. Nature 1995; 378: 206-208.

12. Montes de Oca Luna R, Wagner DS, Lozano G. Rescue of early embryonic lethality in mdm2-deficient mice by deletion of p53. Nature 1995; 378: 203-206.

13. Chavez-Reyes A, Parant JM, Amelse LL, de Oca Luna RM, Korsmeyer SJ, Lozano G. Switching mechanisms of cell death in mdm2- and mdm4-null mice by deletion of p53 downstream targets. Cancer Res 2003; 63: 8664-8669.

14. Mendrysa SM, McElwee MK, Michalowski J, O'Leary KA, Young KM, Perry ME. mdm2 is critical for inhibition of p53 during lymphopoiesis and the response to ionizing irradiation. Mol Cell Biol 2003; 23: 462-472.

15. Grier JD, Xiong S, Elizondo-Fraire AC, Parant JM, Lozano G. Tissue-specific differences of p53 inhibition by Mdm2 and Mdm4. Mol Cell Biol 2006; 26: 192-198.

16. Francoz S, Froment $P$, Bogaerts S, De Clercq S, Maetens M, Doumont G et al. Mdm4 and $\mathrm{Mdm} 2$ cooperate to inhibit p53 activity in proliferating and quiescent cells in vivo. Proc Nat Acad Sci USA 2006; 103: 3232-3237.

17. Xiong S, Van Pelt CS, Elizondo-Fraire AC, Liu G, Lozano G. Synergistic roles of Mdm2 and Mdm4 for p53 inhibition in central nervous system development. Proc Natl Acad Sci USA 2006; 103: 3226-3231.

18. Boesten LS, Zadelaar SM, De Clercq S, Francoz S, van Nieuwkoop A, Biessen EA et al. Mdm2, but not Mdm4, protects terminally differentiated smooth muscle cells from p53-mediated caspase-3-independent cell death. Cell Death Differ 2006; 13: 2089-2098.

19. Lengner CJ, Steinman HA, Gagnon J, Smith TW, Henderson JE, Kream BE et al. Osteoblast differentiation and skeletal development are regulated by Mdm2-p53 signaling. J Cell Biol 2006; 172: 909-921. 
20. Sancho $E$, Batlle $E$, Clevers $H$. Signaling pathways in intestinal development and cancer Annu Rev Cell Dev Biol 2004; 20: 695-723.

21. Crosnier C, Stamataki D, Lewis J. Organizing cell renewal in the intestine: stem cells, signals and combinatorial control. Nat Rev Genet 2006; 7: 349-359.

22. Brittan M, Wright NA. Stem cell in gastrointestinal structure and neoplastic development. Gut 2004; 53: 899-910.

23. Clarke AR, Gledhill S, Hooper ML, Bird CC, Wyllie AH. p53 dependence of early apoptotic and proliferative responses within the mouse intestinal epithelium following gammairradiation. Oncogene 1994; 9: 1767-1773.

24. Merritt AJ, Potten CS, Kemp CJ, Hickman JA, Balmain A, Lane DP et al. The role of p53 in spontaneous and radiation-induced apoptosis in the gastrointestinal tract of normal and p53-deficient mice. Cancer Res 1994; 54: 614-617.

25. Wilson JW, Pritchard DM, Hickman JA, Potten CS. Radiation-induced p53 and p21WAF-1/ CIP1 expression in the murine intestinal epithelium: apoptosis and cell cycle arrest. Am J Pathol 1998; 153: 899-909.

26. Grier JD, Yan W, Lozano G. Conditional allele of mdm2 which encodes a p53 inhibitor Genesis 2002; 32: 145-147.

27. Madison BB, Dunbar L, Qiao XT, Braunstein K, Braunstein E, Gumucio DL. Cis elements of the villin gene control expression in restricted domains of the vertical (crypt) and horizontal (duodenum, cecum) axes of the intestine. J Biol Chem 2002; 277: 33275-33283.

28. Soriano P. Generalized lacZ expression with the ROSA26 Cre reporter strain. Nat Gene 1999; 21: 70-71.

29. Clarke RM. The effect of growth and of fasting on the number of villi and crypts in the small intestine of the albino rat. J Anat 1972; 112 (Part 1): 27-33.

30. Potten CS. Radiation, the ideal cytotoxic agent for studying the cell biology of tissues such as the small intestine. Radiat Res 2004; 161: 123-136.

31. Potten CS, Booth C, Tudor GL, Booth D, Brady G, Hurley P et al. Identification of a putative intestinal stem cell and early lineage marker; musashi-1. Differentiation 2003; 71: 28-41.
32. Kayahara $\mathrm{T}$, Sawada M, Takaishi S, Fukui $\mathrm{H}$, Seno $\mathrm{H}$, Fukuzawa $\mathrm{H}$ et al. Candidate markers for stem and early progenitor cells, Musashi-1 and Hes1, are expressed in crypt base columnar cells of mouse small intestine. FEBS Lett 2003; 535: 131-135.

33. Clevers H. Wnt/beta-catenin signaling in development and disease. Cell 2006; 127: 469-480.

34. Wielenga VJ, Smits R, Korinek V, Smit L, Kielman M, Fodde R et al. Expression of CD44 in Apc and Tcf mutant mice implies regulation by the WNT pathway. Am J Pathol 1999; 154: 515-523.

35. Sanderson MP, Dempsey PJ, Dunbar AJ. Control of ErbB signaling through metalloprotease mediated ectodomain shedding of EGF-like factors. Growth Factors 2006; 24: 121-136.

36. Hall PA, Coates PJ, Ansari B, Hopwood D. Regulation of cell number in the mammalian gastrointestinal tract: the importance of apoptosis. J Cell Sci 1994; 107 (Part 12): 3569-3577.

37. O'Leary KA, Mendrysa SM, Vaccaro A, Perry ME. Mdm2 regulates p53 independently of p19(ARF) in homeostatic tissues. Mol Cell Biol 2004; 24: 186-191.

38. Ringshausen I, O'Shea CC, Finch AJ, Swigart LB, Evan GI. Mdm2 is critically and continuously required to suppress lethal p53 activity in vivo. Cancer Cell 2006; 10: 501-514.

39. Muncan V, Sansom OJ, Tertoolen L, Phesse TJ, Begthel H, Sancho E et al. Rapid loss of intestinal crypts upon conditional deletion of the Wnt/Tcf-4 target gene c-Myc. Mol Cell Biol 2006; 26: 8418-8426

40. Cummins AG, Jones BJ, Thompson FM. Postnatal epithelial growth of the small intestine in the rat occurs by both crypt fission and crypt hyperplasia. Dig Dis Sci 2006; 51: 718-723.

41. Korinek V, Barker N, Moerer P, van Donselaar E, Huls G, Peters PJ et al. Depletion of epithelial stem-cell compartments in the small intestine of mice lacking Tcf-4. Nat Genet 1998; 19: 379-383.

42. Yarden $\mathrm{Y}$, Sliwkowski MX. Untangling the ErbB signalling network. Nat Rev Mol Cell Biol 2001; 2: 127-137.

Supplementary Information accompanies the paper on Cell Death and Differentiation website (http://www.nature.com/cdd) 